W.W. Shane and J. Bystedt

Leiden Observatory

If we view spiral structure in galaxies as a manifestation of some dynamical process which we wish to understand, then the observer can distinguish two facets of the problems. In the first place he must consider the observable consequences suggested by theoretical developments. In the second place he must endeavour to interpret the available observations in terms of realistic physical models, where such models may require detailed information beyond that required for an understanding of spiral structure as a dynamical phenomenon. We shall consider these two aspects in turn.

\title{
1. OBSERVATIONS AND THEORY
}

Looking back about three years, as we often do in reviewing developments in a field such as this, some of us recall the conference on spiral galaxies held in Bures-sur-Yvette (Weliachew, 1975). The conference was timely and the general tone optimistic. The questions which had been raised about the relevance of the density-wave mechanism to spiral structure (e.g. Piddington, 1973) had been answered adequately (Bok and Bok, 1974) and the required next steps seemed to be clear enough. It is true that most of the problems that are troubling us now were already with us then, but their significance was probably not clear to most observers. At the time we were mainly concerned with identifying the density wave (for example, through matching the velocity and density patterns in a specific mode1, e.g. Visser, 1975) and with determining the density-wave parameters which best fit the observations of a specific galaxy (e.g. Rots, 1975; Guibert, 1975). The first attempts to do this had been reasonably promising (Shu et a1., 1971) and we seemed to need only observations with a slightly more favorable combination of resolution and sensitivity to do the job properly.

It now appears that this view was far too simple. The theoretical difficulties have remained with us, despite many valuable suggestions for their solution, and have become more pressing and more evident to the observers. At the same time we saw that the observational data re- 
quired for an attack upon these problems were much less easily accessable than we might have wished. We can list some of the major questions, without attempting to discuss their theoretical implications.

1. Stability problems. In order that the disk be protected against large-scale instabilities, Ostriker and Peebles (1973) have postulated a massive halo whose existance cannot easily be confirmed through direct observations. On the other hand, in order that the spiral structure may survive, the disk must be unstable with respect to certain spiral modes, implying an upper limit to the admissible velocity disperisons of the disk stars (Lau and Bertin, this symposium). Observational determinations of these velocity dispersions are, except in the Galaxy, beyond our current capabilities.

2. Propagation, maintenance and regeneration of spiral waves. There has been no lack of suggestions as to the means by which the spiral pattern can be maintained or regenerated. Both internal and external processes may play a role. The reflection and amplification mechanism suggested by Lin and his coworkers (e.g. Lin, 1975) appears promising but a detailed study of the resonance regions is required, whereas one of the long-standing observational difficulties has been the very identification of resonance regions in galaxies (Oort, 1975). The suggestion of tidal excitation of spiral waves has acquired new meaning through the realization that, aside from the perturbing effects of companions in some systems so convincingly illustrated by Toomre and Toomre (1972), many galaxies may be disturbed by massive infalling intergalactic gas clouds whose individual identities are hard to establish (Gunn, 1977).

3. Simultaneous presence of several spiral modes. Although this also is not a new suggestion (Lin, 1967), it has become increasingly clear that spiral patterns in galaxies can seldom be represented by a single spiral mode (Bertin et a1., 1977), nor is there any theoretical objection to the simultaneous presence of several modes with comparable amplitude. Unfortunately this possibility introduces so many free parameters into any proposed model that fitting such a model to the observations becomes a rather unrewarding exercise in which it becomes possible to fit any conceivable set of observations through a suitable choice of parameters. In the absence of additional observational constraints it is not entirely clear how we should proceed further along this line.

Meanwhile improved instruments have not rewarded the observers, as some of us had hoped, with a clearer picture of spiral structure. Rather they have revealed a baffling complexity in the structure of galaxies which threatens to frustrate any attempt at a simple interpretation. Many of these complexities, in fact, have long been evident. Three examples will illustrate some of the problems.

M51 is a beautiful spiral (the first nebula whose spiral morphology was recognized) and has been studied in great detail. The arms are well defined in $\mathrm{H}$ I, H II, dust and non-thermal continuum and their relative positions have been analyzed in terms of a density wave with gratifying results. But who, in view of the analysis by Toomre and Toomre (1972) believes that the galaxy would look the same in the absence of its com- 
panion? And why is it that, despite the apparent symmetry, the optical major axis departs by at least $45^{\circ}$ from the kinematically determined line of nodes?

M101, on the other hand, is strongly asymmetric in appearance without there being any prominent companion which might help to account for this. It has been studied in detail in the $21-\mathrm{cm}$ line (Allen et a1. 1973) and radio continuum (Israel et al., 1975) and, although very important results have been derived, it remains an unattractive object for the testing of dynamical theories.

M31 appears to possess we11-defined spiral arms, although the high inclination angle obscures the pattern. Nevertheless, the arms in the inner region, where most spiral galaxies exhibit the simplest structure, show large radial motions (Shane, this symposium) reminiscent of those detected in the Milky Way. No simple density-wave model can account for these motions.

One nearby galaxy does seem to behave as one might expect from a simple density-wave mode1, and this is M81. Perhaps this should surprise us since M81 is surrounded by companions and intergalactic gas clouds and even has a peculiar nucleus. Nevertheless, with all of the reserve that is appropriate in considering a unique case, this seems to offer us our best opportunity to test a density-wave model. Herman Visser of the Kapteyn Laboratory in Groningen has done this in a study which represents the state of the art in fitting density-wave models to galaxies (Visser, this symposium). Perhaps the most difficult decision to be made is the degree of complexity of the model to be examined. On the one hand, it must be realistic, within the limits of the observations, and on the other, it must not possess so may degrees of freedom as to make a fit meaningless. Visser's results suggest that he has reached a suitable compromise in this respect.

It would be wrong to conclude even a brief discussion of model fitting without mentioning the particular problems of barred spirals. Here the theoretical analysis (Sanders and Huntley, 1976) has not progressed far beyond calculations of the response of a gas to an imposed gravitational perturbation. A self-consistent solution has not yet appeared. The calculations predict pronounced gas streaming parallel to the bar with shocks approximately in the position of the dust lanes. Direct observational confirmation is difficult because the most conspicuous barred spirals will have the bar close to the plane of the sky and the streaming velocities will be unobservable. In such systems a less direct check is possible by examining the behavior of the apparent rotation velocity inside and outside the barred region. The elongated gas orbits in the inner region should result in a measured rotational velocity in the inner (but not the outer) part substantially below that required for circular rotation. Work in progress on NGC 1300 and 4236 does not seem to indicate this but the results are verv preliminary. Much more suggestive are the radio (Sancisi, private communication) and 
optical observations of NGC 5383 (Peterson et a1., 1977), a barred spiral whose bar may well be quite inclined with respect to the plane of the sky. The observations here are mutually consistent and, although the interpretation is still open to lively discussion, some evidence for streaming parallel to the bar, as predicted by the calculations, seems to be present. It appears probable that we can expect important developments in the study of barred spirals in the near future.

\section{INTERPRETATION OF THE OBSERVATIONS}

If we are to make proper use of the available observational material we must look carefully at the interpretation. In this section we will discuss some of the more urgent problems.

Van der Kruit (this Symposium) has already discussed in detail the problem of separation of thermal and non-thermal contributions. Attempts to estimate the thermal contribution from optical data are fraught with difficulties. Once the individual color excesses of the $\mathrm{H}$ II regions have been determined, no mean task in itself, and converted into extinctions, the problem remains of accounting for the non-uniformity of the extinction over (and within) the H II region. Application of standard formulae will always lead to an underestimate of the total extinction. Van der Kruit's discussion of M51 (v.d. Kruit, 1977) suggests that this may amount to about $0.5 \mathrm{mag}$. in that particular galaxy, and selection effects (heavily obscured H II regions will be optically too faint to be included) may increase this. In view of these and other difficulties we heartily subscribe to van der Laan's comment (this Symposium) to the effect that there is no satisfactory alternative to observations at several short wavelengths $(<10 \mathrm{~cm})$ as a means of isolating the thermal contribution.

Understanding the non-thermal radio radiation also requires a knowledge of the physical conditions beyond our capability of direct observation. The wel1-known expression for volume emissivity is $\varepsilon \propto \rho_{e} B^{1-\alpha}$. Since both the density of relativistic electrons, $\rho_{e}$, and the magnetic field strength, $B$, are (in some models) proportional to the gas density $\rho$, we often write $\varepsilon \propto \rho^{2-\alpha}$ (Mathewson et a1., 1972) and, replacing both $\varepsilon$ and $\rho$ by their volume averages, derive the useful, if incorrect, relation, $\langle\varepsilon\rangle \propto\langle\rho\rangle^{2-\alpha}(!)$. The situation is further complicated when we realize that the mixture of physical conditions in regions of different average density will be quite dissimilar, as will be the degree of inhomogeneity. It would seem that further progress in interpreting the non-thermal continuum radiation from galaxies will depend upon a better understanding of the local physical conditions.

Even interpretation of the line radiation is subject to uncertainties. It is customary to accept the integrated brightness temperature as a measure of the column density of gas. Two considerations throw doubt upon the validity of this assumption: In the first place, although the measured brightness temperature is never large enough to suggest 
significant self-absorption, beam dilution makes it quite possible that it is important on a small scale so that considerable H I may be hiding in or behind dense clouds (e.g. Shane, 1971). In the second place it is not clear that H I need be conserved. Molecule formation may play a significant role in the spiral arms as may ionization in large regions of low density between the arms. Happily, Visser has shown that the observations of M81 can be interpreted on the basis of the simplest assumptions without introducing serious inconsistencies, but we should remain on guard.

One of the often quoted quantities in discussions of spiral galaxies is the arm to inter-arm ratio of continuum radiation intensity (e.g. Segalovitz, 1976). It is taken as an indication of the degree of compression of the gas in the spiral arms and is required for the separation of the spiral arm contributions from the base disk (Mathewson et a1. 1972). But the quantity determined from the observations does not always represent what one would wish. The peak represents an average taken over a resolution element and would be higher if the resolution was better, and it also varies eratically along the arm, whereas the minimum, or even the average, between arms is usually close to or below the sensitivity limit of the instrument. Thus what is often quoted is the ratio of peak to the mean over a circle in the galaxy. This is quite a different quantity and has more to do with the width and profile of the arm than with the interarm values. This should be kept in mind when interpreting these results.

One of the conclusions which has been drawn from analysis of this kind of data is that the enhancement of continuum radiation in the arm does not exceed that of line radiation by as much as one would be lead to believe from the volume emissivity relation quoted above (Shane, 1975), at least when one considers a simple two-dimensional mode1 (Mathewson et a1. 1972). The solution has been sought in the escape of relativistic plasma from the arms through Parker instabilities (Mouschovias et a1. 1974) and it is clear that this offers a promising means of maintaining a halo or thick disk in the non-thermal continuum (e.g. Levy, this symposium) which, among other things, will reduce the arm to inter-arm ratio. Less clear is it that the Parker instability can account for the "beads on a string" appearance of spiral arms. Given the usual length $\left(1 \mathrm{kpc}\right.$ ) and time ( $10^{8}$ years) scales, bulk gas velocities approaching $10 \mathrm{~km} / \mathrm{s}$ are required if the beads are to be formed before passing out of the string. Such velocities should be observable using new instruments. What is not evident is how the magnetic field, conspiring with the gravitational potential of the disk, can provide the required acceleration on such a scale. More detailed calculations are required. Meanwhile the observations, which first seemed to indicate the expected periodic clumping in the H I (Oort, 1974), are, with improved resolution, showing a more chaotic picture in which the beads are a good deal less apparent (Rots and Shane, 1975).

Finally, two relatively new sorts of observations should be mentioned, whose interpretation ought to lead to more insights into the 
nature of spiral arms. After the detection of CO in external galaxies (Rickard et a1. 1977) we have entered the mapping stage, and the first results (Combes et al. 1977a, b) are as encouraging as they are impressive. If we may adopt the suggestion (Bash and Peters, 1976) that CO clouds represent a population with a maximum age of about $3 \times 10^{7}$ years, then the rules of star migration (e.g. Wielen, this Symposium), will apply equally to CO clouds, while we will enjoy the additional advantage of good velocity data. Thus radio astronomers will enter a field which has thus far been virtually the monopoly of our optical colleagues.

The first radio polarization map of a spiral galaxy has been produced (Segalovitz et a1. 1976) and, although the galaxy observed, M51, is in many ways atypical, a preliminary attempt at interpretation is possible. The observations were discussed in terms of a model in which a fraction $f$ of the volume of the galaxy is occupied by a uniform magnetic field (with azimuthal orientation) and the remainder by a randomly oriented field. Taking account of a substantial thermal contribution (which was not done in the original discussion), we can estimate $f=0.2$. In a quiescent galaxy the magnetic field will be drawn out into uniform rings by differential rotation. The time required for this might be estimated as about $3 A \approx 5 \times 10^{7}$ years (adopting galactic values for convenience). We call this the combing time, $t$, following a suggestion by Toomre. But from time to time the magnetic field is randomized by a mixing event. A simple calculation serves to specify the ratio of this mixing time, $t_{\mathrm{m}}$, to $t_{\mathrm{c}}$ in terms of $f$, and we find that $f=0.2$ requires $t_{\mathrm{m}}=0.4 t_{\mathrm{c}}^{\mathrm{m}}=2 \times \mathrm{x}^{\mathrm{c}} 10^{7}$ years. Supposing that these mixing events are supernova explosions and that supernovae occur once per 50 years 3 (Katgert and Oort, 1967) and are distributed over a volume of $200 \mathrm{kpc}^{3}$ (again adopting galactic estimates) we may deduce as characteristic radius of action of a single supernova explosion about 50 pc, a not unreasonable value. But clearly a more realistic model is required, taking into account the very different conditions in and between the arms, before serious calculations can be considered. Nevertheless, we hope this small example will serve to emphasize the need for more polarization observations at wavelengths of $6 \mathrm{~cm}$ or less.

\section{REFERENCES}

Allen, R.J., Goss, W.M., and van Woerden, H.: 1973, Astron. Astrophys. $29,447$.

Bash, F.N. and Peters, W.L.: 1976, Astrophys. J. 205, 786.

Bertin, G., Lau, Y.Y., Lin, C.C., Mark, J.W-K., and Sugiyama, L.: 1977, submitted to Astrophys. J. Letters.

Bok, B.J. and Bok, P.F.: 1974, The Milky Way, Harvard Univ. Press, 4 th ed., p. 228.

Combes, F., Encrenaz, P.J., Lucas, R., and Weliachew, L.: 1977a, Astron. Astrophys. 55, 311 .

Combes, F., Encrenaz, P.J., Lucas, R., and Weliachew, L.: 1977b, submitted to Astron. Astrophys. 
Guibert, J.: 1975, in Weliachew 1975, p. 263.

Gunn, J.E.: 1977, in B.M. Tinsley and R.B. Larson (eds.), The Evolution of Galaxies and Stellar Populations, Yale Univ. Observatory, p. 445.

Israe1, F.P., Goss, W.M. and Allen, R.J.: 1975, Astron. Astrophys. 40, 421.

Katgert, P. and Oort, J.H.: 1967, BuZZ. Astr. Inst. Netherl. 19, 239.

Kruit, P.C. van der: 1977, Astron. Astrophys. 59, 359.

Lin, C.C.: 1967, I.A.U. Symposium No. 31, 313.

Lin, C.C.: 1975, in Weliachew 1975, p. 493.

Mathewson, D.S., Kruit, P.C. van der and Brouw, W.N.: 1972, Astron. Astrophys. $17,468$.

Mouschovias, T.Ch., Shu, F.H. and Woodward, P.: 1974, Astron. Astrophys. $33,73$.

Oort, J.H.: 1974, in Galaxies and Relativistic Astrophysics (B. Barbanis and J.D. Hadjidemetriou, eds.), Springer Verlag, p. 1.

Oort, J.H.: 1975, in Weliachew 1975, p. 533.

Ostriker, J.P. and Peebles, P.J.E.: 1973, Astrophys. J. 186, 467.

Peterson, C.J., Rubin, V.C., Ford, W.K.Jr. and Thonnard, N.: 1977 , BulZ. Am. Astron. Soc. 9, 336.

Piddington, J.H.: 1973, Astrophys. J. 179, 755.

Rickard, L.J., Palmer, P., Morris, M., Zuckerman, B. and Turner, B.E.: 1975, Astrophys. J. Letters 199, 75.

Rots, A.H.: 1975, in Weliachew 1975, p. 201.

Rots, A.H. and Shane, W.W.: 1975, Astron. Astrophys. 45, 25.

Sanders, R.H. and Huntley, J.M.: 1976, Astrophys. J. 209, 53.

Segalovitz, A.: 1976, Astron. Astrophys. 52, 167.

Segalovitz, A., Shane, W.W. and de Bruyn, A.G.: 1976, Nature 264, 222.

Shane,W.W.: 1971 , Astron. Astrophys. Suppl. 4, 315.

Shane, W.W.: 1975, in Weliachew 1975, p. 217.

Shu, F.H., Stachnik, R.V. and Yost, J.C.: 1971, Astrophys. J. 166, 465. Toomre, A. and Toomre, J.: 1972, Astrophys. J. 178, 623.

Visser, H.C.D.: 1975, in Weliachew 1975, p. 211.

Weliachew, L. (ed.): 1975, La Dynamique des Galaxies Spirales, CNRS, Paris.

DISCUSSION FOLLOWING REVIEW II.2 GIVEN BY W.W. SHANE

LEVY: I would like to comment on some questions raised by Dr. Shane concerning the Parker instabilities in the disk.

(1) Observability of streaming motions of the gas: The present state of the gas in our galaxy or in other galaxies can best be described as a state that exists after the full development of the instability. So while one might occasionally expect to see some streaming motion, in gas accumulations that have existed for a sufficiently long period of time one wouldn't expect to see much gas streaming anymore.

(2) Availability of energy to drive the instability: The gravitational energy of the stellar disk, which predominates, and the magnetic energy are sufficient. The time scale for the growth of the instability is the free-fall time for the material in the rotational field, so that there is automatically enough force to drive the instability. 
(3) Material is never lifted! The instability is the result of material sliding down the magnetic field lines, not of material being lifted. So you need not account for lifting material up any height at a11. 